\title{
On 3 to 6 year cycles in the time of geomagnetic storm sudden commencement occurrence and ENSO climate cycles
}

\author{
G. Ja. Khachikjan ${ }^{1}$ and G. J. Sofko ${ }^{2}$ \\ ${ }^{1}$ Institute of Seismology, 480060, Al-Farabi, 75 a, Almaty, Kazakhstan \\ ${ }^{2}$ Institute of Space and Atmospheric Studies, University of Saskatchewan, S7N 5E2 Saskatoon, Canada \\ Received: 7 July 2005 - Revised: 9 October 2005 - Accepted: 28 October 2005 - Published: 9 January 2006
}

\begin{abstract}
The geographic longitude of the subsolar meridian (SML) evaluated at the time of geomagnetic Storm Sudden Commencement (SSC) occurrence shows an unexpected organized longitudinal progression with time, for successive SSC events. The progression includes, in particular, quasi-periodic cycles of about 3 to more than 6 year duration which show a close relationship with the sunspot cycles. The 3 to 6 year SML cycles also show a surprising agreement with the naturally occurring climate cycles known as El Nino/Southern Oscillations (ENSO). On the basis of SSC and El Niño records from 1968 to 2003, it is found that a warm ENSO phase (El Niño) takes place at about the middle of each of seven identified SML cycles.
\end{abstract}

\section{Introduction}

The geomagnetic field intensity responds to a Sudden Impulses (SI) simultaneously all over the world (Ferraro at al., 1951; Jackson, 1952). Generally, an SI event is caused by a sudden increases in the dynamic pressure of the solar wind near the Earth's orbit. At the time of an SI, there is often an interplanetary shock wave in the solar wind, whose disturbed state is frequently associated with a coronal mass ejection (Lindsay et al., 1994). The magnetic disturbance associated with the SI propagates to the surface of the earth and is recorded as sharp changes in the ground magnetograms. It was found by Siscoe et al. (1968), Su and Konradi (1975), and Russell et al. (1994a, b) that the ground level magnetic field (nT) increase is proportional to the square root of the dynamic pressure $\left(\mathrm{nPa}^{1 / 2}\right)$ with a coefficient that ranges from

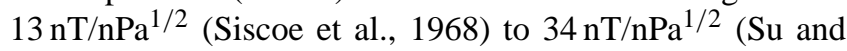
Konradi, 1975). In the definition introduced by Mayaud (1973), an SI event which is followed by a magnetic storm or by an increase in geomagnetic activity lasting at least one hour is named a Storm Sudden Commencement (SSC). Evi-

Correspondence to: G. Ja. Khachikjan

(kha@kaznet.kz) dently, the SSC onset marks the time of a dynamic increase in the interaction of the solar wind with the magnetosphere, with a resulting strong penetration of solar wind momentum and energy into the Earth's environment. At present, records of SSC occurrence are provided by Observatorio del Ebro, Roquetes, Spain, on behalf of the IAGA Service on Rapid Magnetic Variations. The routine procedure of the Service in establishing the list of SSCs consists of several steps, following the instructions given by Mayaud (1973). Using monthly reports of rapid variations prepared by 43 magnetic observatories, a combined list of all events is compiled. This list is then checked against the magnetograms of two low latitude observatories; cases that are reported by only a very few observatories and that do not have a clear SSC signature in the magnetograms of two low latitude observatories are eliminated. All other cases are then included in a new list, and copies of the magnetograms are obtained from the five collaborating low latitude observatories that are regularly spaced in longitude, or from their supplementary observatories. From the magnetograms of these five observatories, every SSC is evaluated independently for each observatory and to each event a code number ( 0 to 3 ) is assigned, the value depending upon the clarity with which the event can be identified as an SSC. If an event is characterized by three code " 0 " numbers out of the five observatories, it is rejected from the list. Thus, the final SSC list is one that is suitable for scientific analysis. It can be found at the (ftp://ftp.ngdc.noaa.gov/ STP/SOLAR_DATA/sudden_commencements/storm2.SSC).

\section{Results}

\subsection{The non-random occurrence of SSC times}

It is usually considered that SSC occurrence times are random because they depend on the solar wind state only (Mayaud, 1975). However, it was found recently by Khachikjan et al. (2005) that SSC times are not in random order but rather are organized according to universal time, 


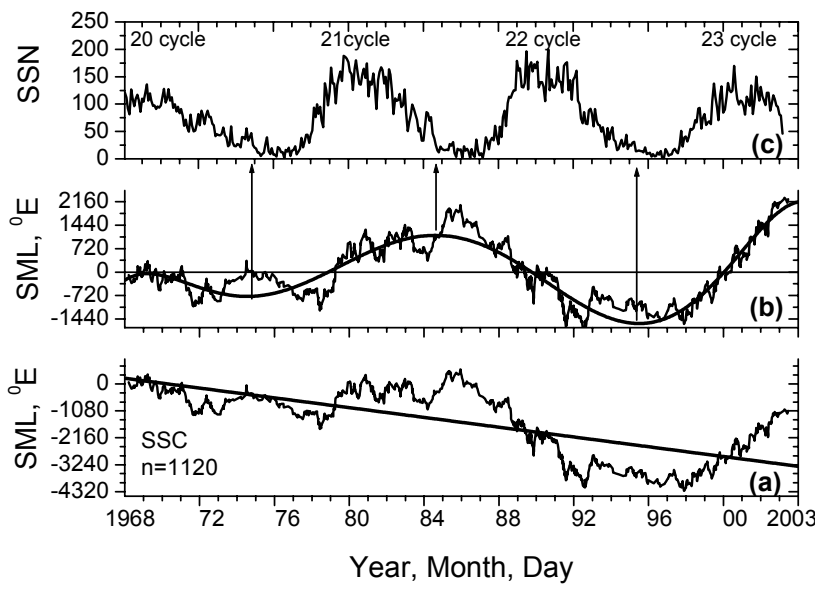

Fig. 1. Geographic longitude of the subsolar meridian (SML) at the time of 1120 SSCs from 14 March 1968 to 18 June 2003 in comparison with monthly sunspot numbers (Khachikjan et al., 2005). (a) the SML progression (thin) and its linear westward trend (thick); (b) the SML progression after eliminating the linear trend (thin line), its fit to a polynomial of order $n=6$ is shown by the thick line; (c) monthly sunspot numbers (SSN) for solar cycles 20-23.

season, and the 22-year period of the solar magnetic cycle. In the above study of SSC behavior, the geographic longitude of the subsolar meridian (SML) was evaluated at the time of each of the 1120 SSCs that occurred during the 35-year interval from 1968 to 2003, and then the progression of that meridian with time was analyzed. To obtain such a progression, sequential SML values were connected, using the shortest longitudinal distance between successive SSC events. It was found that, on the average, the absolute value of the longitudinal angular separation between sequential SML values was equal to about 90 degrees, but that it varied from about 1 to almost 180 degrees. At the same time, no longitudinal distance was found that was exactly 180 degrees, and only for one sequential pair it was very close to that value $\left(179.75^{\circ}\right.$ on 4 July 1991 for SSC events that occurred at 03:37 and 15:36 UT). Thus, from a mathematical point of view, it was not difficult to evaluate reliably the direction (westward or eastward) of the progression of SML positions.

Figure 1 shows the geographic longitude of the subsolar meridian (SML) at the time of 1120 SSCs from 14 March 1968 to 18 June 2003 in comparison with monthly sunspot numbers (Khachikjan et al., 2005). In Fig. 1a, the raw SML progression (thin) and its linear westward trend (thick) are shown. After elimination of this linear trend, a 22-year variation is seen in the longitudinal location of the subsolar meridian (Fig. 1b).

2.2 On the reliability of 22-year variation in the SML position

Comparison of the 22-year SML variation (1b) with sunspot numbers (1c) shows that during odd-numbered solar cycles 21 and 23, the SML values drifted mainly eastward, whereas

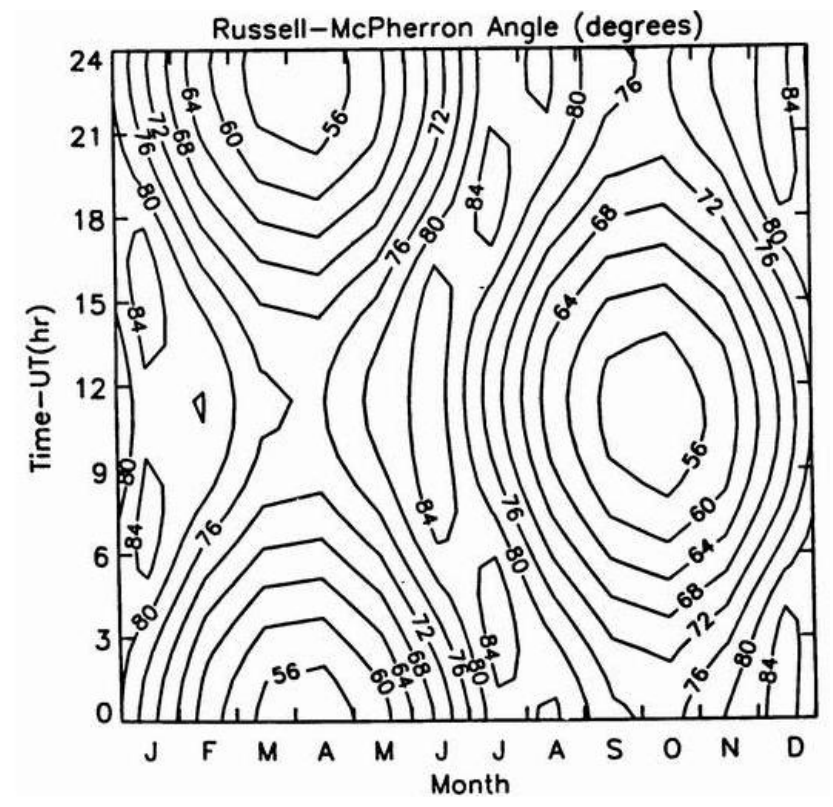

Fig. 2. UT/seasonal variations of the angle $\varphi$, controlling parameter in the Russell-McPherron mechanism, according to the data of Cliver et al. (2000).

they drifted mainly westward during even-numbered solar cycles 20 and 22. This result allows one to suggest that the 22-year SML variation is related to the 22-year magnetic solar cycle. We consider that this is in agreement with the prediction by Russell and McPherron (1973) who suggested that the time of effective interaction of the solar wind with the magnetosphere that is followed by increasing of geomagnetic activity is controlled by the interplanetary magnetic field (IMF) polarity. In the model by Russell and McPherron it is assumed that for the typical IMF "Parker spiral" configuration, geomagnetic activity maximizes when the southward component in the GSM (geocentric solar magnetospheric) system maximizes. In the southward component model the controlling parameter is the angle $\varphi$ measured in the $y-z$ (GSM) plane between the $\mathrm{z}$-axis of the GSM coordinate system and the solar equatorial plane. This angle (Fig. 2) varies over a range from about $52^{\circ}$ at the equinoxes to $90^{\circ}$ at the solstices. When the angle $\varphi$ reaches its minimum value of $52^{\circ}$, that occurs on 5 April at 22:30 UT and on 8 October at 10:30 UT, geomagnetic activity is expected to reach a maximum, because at these times a solar wind magnetic field lying entirely in the Sun's equatorial plane has its maximum projection on the z-axis of the GSM coordinate system. In their southward component model, Russell and McPherron (1973) suggest that the spring maximum in geomagnetic activity at 22:30 UT is associated on the average with negative IMF polarity (directed toward the sun) and that the fall maximum at 10:30 UT is associated with positive IMF polarity (directed away from the sun).

Figure 2 demonstrates that the time of effective solar windmagnetosphere interaction should constantly change in the course of year from 22:30 UT in the spring to 10:30 UT in 


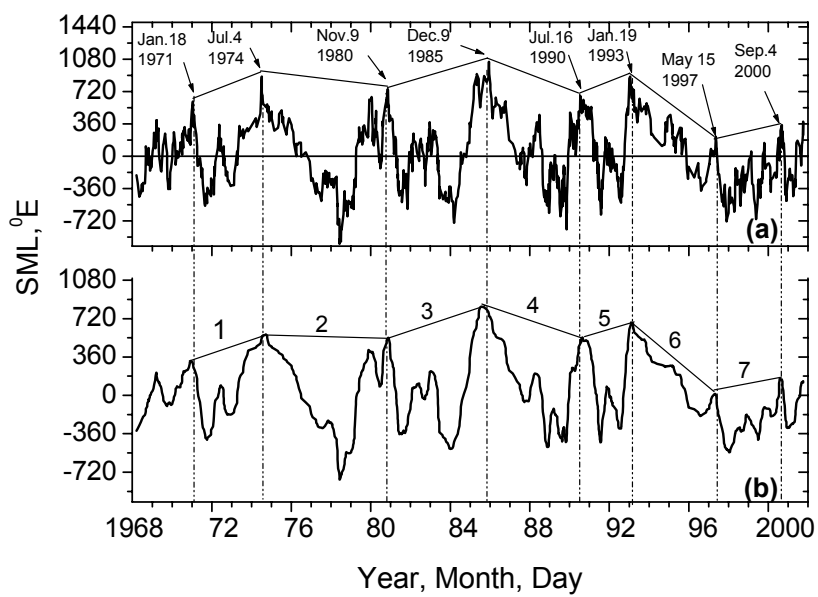

Fig. 3. The geographic longitude of the subsolar meridian (SML) at the time of 1120 SSCs from 14 March 1968 to 18 June 2003, after elimination of the westward linear trend and the 22-year variation (a), and after 15-point smoothing (b).

the fall, and then from 10:30 UT in the fall to 22:30 UT in the spring. This motion may occur in both the clockwise and counterclockwise directions, so the subsolar meridian corresponding to Universal Times for the SSC events show westward and eastward progressions, respectively. Evidently, the preferred direction of that progression should be modulated by the IMF polarity variations because the spring and fall maxima occur preferentially for negative (toward the sun) and positive (away from the sun) polarities, respectively. Since the IMF polarity has a 22-year periodicity, one could assume that a similar periodicity should also be observed in the time of occurrence of consecutive SSCs and in the SML position. On the basis of SSC and IMF records from 1968 to 2003, it was found by Khachikjan et al. (2005) that the 22year variation in the SML position is in close agreement with the 22-year variation in the interplanetary magnetic field polarity. After elimination of the 22-year variation of the SML position, other shorter-period variations from about 3 to more then 6 years in the SLM progression were revealed (Fig. 3).

2.3 On the reliability of short-time variations in the SML position

In Fig. 3a the progression with time of the geographic longitude of the subsolar meridian (SML) at the time of 1120 SSCs for the 35 years from 14 March 1968 to 18 June 2003 is shown, after the elimination of both the linear trend and the 22 -year variation, as discussed above. Figure $3 \mathrm{~b}$ shows the SML progression smoothed by a 15 -point running average.

Dash-dotted lines in Fig. 3 denote 7 quasi-periods (cycles), whose duration varies from about 3 to more than 6 years. The SML behavior in each of the seven identified cycles has a similar pattern: from the beginning of the cycle, the SML value moves more or less continually westward with time, then it stops and shows smaller variations, after which it moves eastward more or less continuously and fi-

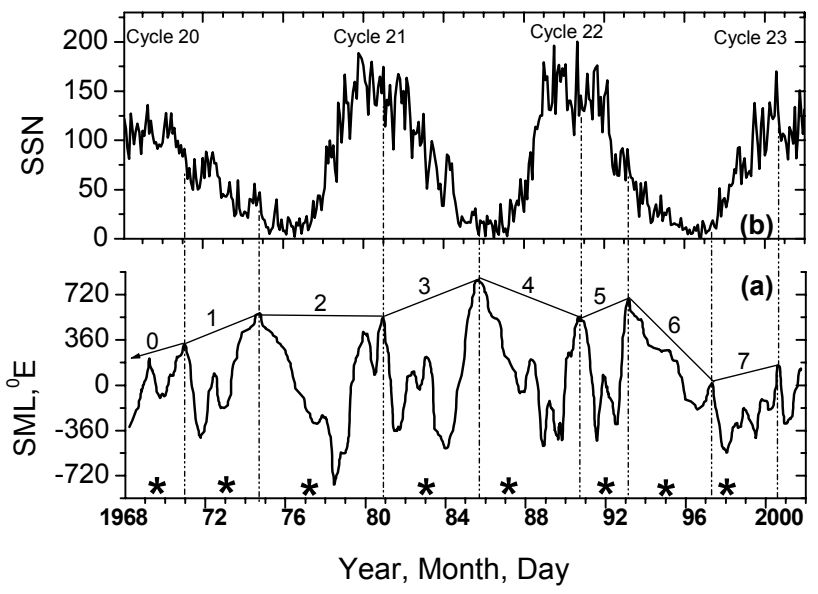

Fig. 4. The geographic longitude of the subsolar meridian (SML) at the time of 1120 SSCs from 14 March 1968 to 18 June 2003 after 15-point SML smoothing (a). The stars at the bottom indicate years with El Niño events. The 3-6 year SML cycles are in agreement with climate ENSO cycles. (b)Monthly sunspot numbers for solar cycles 20 to 23 .

nally reaches an "extreme point" (maximal SML value). After reaching an extreme point, a new SML cycle begins. The dates on which the extreme points occurred are indicated in Fig. 3a. They are as follows: 18 January 1971; 4 July 1974; 9 November 1980; 9 December 1985; 16 July 1990; 19 January 1993; 15 May 1997; 4 September 2000.

The short-period SML variations in Fig. 3 (on the average 2-3 revolutions around the globe) have much smaller amplitude than the long-period variations in Fig. 1a (more then 10 revolutions around the globe). A question arises: are the short-period variations meaningful or merely subjective? To check this, consider Fig. 4, where the short-period SML cycles are compared with 11-year solar cycles. It is seen that occurrence of the 3-6 year SML cycles is roughly coordinated with the ascending and declining phases of the 11-year solar cycle. In particular, the single SML cycles 2,4 , and 7 occurred only during the ascending phase of 11-year solar cycles. The single cycle 3 occurred during the declining phase of odd solar cycle 21. On the other hand, during the declining phases of the two even solar cycles (20 and 22), not one but two SML cycles $(0,1$ and 5,6$)$ occurred. This could have a basis in the observation that during the declining phase of the even-numbered solar cycles, geomagnetic activity is higher than during the declining phase of the oddnumbered solar cycles (Chernosky, 1966).

Thus, the 3-6 year SML cycles identified in Figs. 3 show a close relationship to the ascending and declining phases of the 11-year solar cycle. More over, it is known that the IMF polarity also shows preferred shorter periods during the 22year variation. In particular, Gonzalez and Gonzalez (1987) detected a peak at $\sim 3.7$ year in the IMF polarity variations. Since the SLM progression includes the 22-year periodicity (Fig. 1b) due to the 22-year variation in the IMF polarity (Khachikjan et al., 2005), one could assume that the periodicity of about 3.7 years in the SLM progression is also 

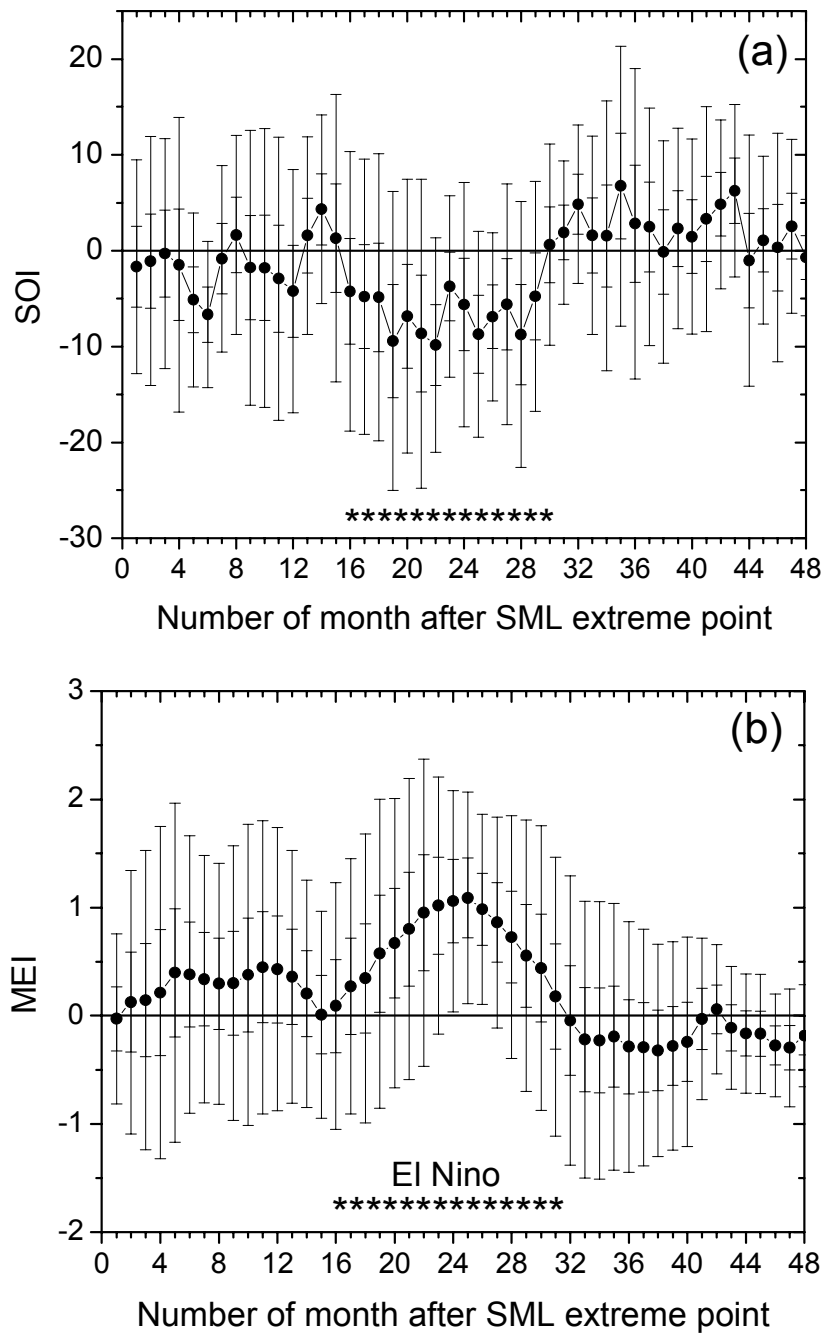

Fig. 5. Monthly SOI (a) and MEI (b) values for seven 48-months periods from the month with the SML extreme point: January 1971, July 1974, November 1980, December 1985, July 1990, January 1993, and May 1997. Wide and narrow bars indicate, respectively, the standard deviation and standard error for averages with percentile 95.0. Stars at the bottoms mark the occurrence period that is typical for the El Nino events.

associated with a 3.7 year variation in the IMF polarity (Gonzalez and Gonzalez, 1987). This may prove the reliability of short-time variations in the SML position.

\subsection{The 3-6 year SML cycles are in agreement with climate} ENSO cycles

Keeping in mind that our planet has a naturally occurring climate cycle known as the El Niño/Southern Oscillation (ENSO), whose duration varies from about 3 to more than 6 years (Ramage, 1986; Quinn et al., 1987), we show with stars in the bottom of Fig. 4 the year of each El Niño event, that occurred during the last three decades: 1969, 1972-1973, 1976-1977, 1982-1983, 1986-1987, 1991-1992, 19941995, 1997-1998 (the latest one, which occurred in 2002-
2003 is not marked in the figure). The duration of the SML cycles varies from about 3 years (16 July 1990-19 January 1993 ) to more than 6 years (4 July 1974-9 November 1980).

The occurrences of both the SML cycles and the El Niño events are rather irregular and the statistical database is limited with only 7 El Niño events presented in Fig. 4. Nevertheless, we made an attempt to determine statistically a mean time lag between the SML extreme point and the El Niño event. For this purpose, the behavior of ENSO-indices (SOI and MEI) was analyzed in relation to the seven SML extreme points indicated in Fig. 3a. The website http://www. bom.gov.au/climate/current/soihtm1.shtml was used for SOI values, while the MEI values were kindly provided by Dr. K. Wolter from the NOAA Climate Prediction Center.

Figure 5 shows monthly SOI (a) and MEI (b) values averaged for seven 48-month periods beginning from the month when the SML extreme point was identified, namely, January 1971 , July 1974 , November 1980 , December 1985 , July 1990, January 1993, and May 1997. Wide and narrow bars indicate, respectively, the standard deviation and standard error for averages with percentile 95.0.

In accordance with the results of Ramage (1986), the typical duration of an El Niño event is about 15 months. Figure 5 really shows $\sim 15$-months duration period (indicated at the bottom with stars) when the SOI values are large and steady negative (5a), and the MEI values are large and steady positive (5b). The above combination of SOI and MEI indices, which is the typical for El Niño event, starts about 15-16 months after the month with the SML extreme point. Thus, one warm ENSO phase (El Niño) takes place within each of 3-6 year SML cycle identified in Fig. 3. On the average, El Niño events occur about 15-16 months after the beginning of the SML cycle.

\section{Discussion}

Beginning from the earliest papers by Hildebrandsson (1897), who noted the global-scale oscillation in surface pressure, Walker (1928), who decided to call it the "Southern Oscillation" (SO), Schell (1965), who related El Niño to variations in the strength of the South Pacific anticyclone and therefore to the SO, and Bjerknes (1966), who described a mechanism of air-sea interaction that linked SO to El Niño, many attempts have been made to link the joint SO and El Niño occurrence with solar variability (Schell, 1956; Berlage, 1957; Mendoza et al., 1991; Kane, 1997; Landscheidt, 1999, and references therein). Some historical steps in this area were overviewed by Mock (1981), who mentioned, in particular: “...Two mechanisms involving variations in solar activity, which might trigger SO, were discussed by Schell (1956): (i) perturbation of the Antarctic circulation due to infusion of corpuscular radiation over the pole, which might lead to a variation in the amount of cold water maintaining the South Pacific High; (ii) expansion of the airmass over the tropical Indian Ocean due to an increase 
in ultraviolet solar radiation, which could increase the high level flow of air into the South Pacific".

The most visible feature of the solar variability is the 11year sunspot cycle. The total solar irradiance varies from year to year with a peak-to peak rms amplitude of about $0.1 \%$ in phase with the 11-year sunspot cycle (Frohlich and Lean, 1998). This variation provides systematic decadal and interdecadal changes in ocean temperatures in phase with the 11-year sunspot cycle (Reid, 1991 and references herein). At the same time, it was quickly recognized that an 11 year variation of the SO and ENSO indices is absent.

Schell (1956) discussed the absence of an 11 year cycle for the SO and suggested the possibility of a breakdown threshold inherent in the nature of the general circulation that would lead to a quasi-periodic readjustment to the slowly varying external forcing provided by the sun. Landscheidt (1999) postulated that the El Niño cycles could be related to solar coronal processes, such as solar eruptions, for which the intensity does not show 11-year cycle, but rather a dual-peak structure during each cycle (Gnevyshev, 1967). Landscheidt (1999) investigated a relationship between El Niño occurrences, solar eruptions and special subsycles in the ascending and declining phases of the 11-year sunspot cycle and found rather similar patterns for the investigated parameters within subcycles. He suggested that a plausible mechanism linking solar eruptions and El Niños could be based on the Forbush decrease (Pudovkin and Veretenenko, 1995; Svensmark and Friis-Christensen, 1997) which shows that, when the solar wind has been strengthened by solar eruptions, the cosmic ray flux weakens leading to a decrease in cloudiness by 2-3 percent lasting a week or longer. He proposed that such short-term effects, especially when they trigger tropical cyclones may release and sustain El Niños. The results in Fig. 4 confirm that El Niño events occur at least twice during each 11-year solar cycle, mainly during the ascending and declining phases. During the declining phase of the even 20th and 22nd solar cycles, when geomagnetic activity is expected to be the strongest (Chernosky, 1966), two El Niños occurred, in 1969; 1972-1973 and 1991-1992; 1994-1995, respectively. This result is in agreement with Mendoza et al. (1991) who investigated a long-range SOI records and noticed that about $63 \%$ of all El Nino events took place during the declining phase of the 11-year sunspot cycle.

El Niño events occur in conjunction with SO, for which the amplitude is depressed during an El Niño event (Ramage, 1986). Thus, to link the SO and El Niño phenomena to solar eruptions (Landscheidt, 1999) or to increased corpuscular and/or ultraviolet solar radiation (Schell, 1956), one needs to show how the solar processes could both produce and destroy the SO that is the regular seasonal changes of longitudinal gradients in air-sea parameters between the east and west parts of the tropical Pacific Ocean. In this connection Mock (1981) noticed, for example: "Schell believed that the observed correlation between sunspot activity and temperature over the tropical Indian Ocean was credible evidence in support of his second mechanism (ii in above). At the same time, why the region around the Indian Ocean should be especially susceptible to solar forcing was not dealt with by Schell". The results in Figs. 3-5, which show agreement between the 3-6 year cycles in the SML progression and El Niño events may provide a hint about how solar variability and space weather could produce and destroy seasonal-longitudinal oscillations in air-sea parameters. Namely, in accordance with the model by Russell and McPherron (1973), the interaction of the solar wind with the magnetosphere is the most effective near the equinoxes and peaks on April 5 at 22:30 UT and on October 8 at 10:30 UT (Fig. 2). An effective interaction event is followed by a rapid and dynamic increase of the particle injection into the Earth's environment (Akasofu, 1981; Nielsen and Honary, 2000). Particle injections are more typical in the predmidnight sector and have a limited longitudinal extent corresponding to the sector occupied by the substorm current wedge (Vagina et al., 1996). Taking this into account, it is not difficult to understand from Fig. 2 that for the most effective interaction time of 22:30 UT in the spring, the predmidnight sector is located in the Europe-Atlantic region, whereas for the most effective interaction time of 10:30 UT in the fall, the predmidnight sector will be located close to the Alaska-Pacific region. Thus, if the air-sea parameters are indeed related somehow to particle injections, the mechanism by Russell and McPherron could explain their regular season-longitudinal oscillations. Moreover, the model by Russell and McPherron (1973) predicts that the spring maximum of interaction is associated mainly with negative IMF polarity, and the fall maximum with positive IMF polarity. Since the IMF polarity varies, one could assume that the time of SSC occurrence, the longitudinal region of particle injection and, consequently, the amplitude of season-longitudinal oscillations in the air-sea parameters will vary with time with the same periods that characterize IMF polarity variations, in particular the $\sim 3.7$ variation noted by Gonzalez and Gonzalez (1987). This could be one reason for the agreement between the periods of geomagnetic SSC and El Niño occurrences. Earlier a similarity in the periodicity ( $\sim 3.5$-years $)$ of the geomagnetic $a a$ index, the southern oscillation index and the sea-surface temperature was recognized by Kane (1997).

In Figure 3a, the last identified SML extreme point is 4 September 2000. In agreement with the results in Fig. 5, one would have expected the next El Niño event to occur about 15-16 months later, near the beginning of 2002, as mentioned Khachikjan and Petelin (2002). In fact, the latest El Niño indeed started in March 2002, about 18 months after the last SML extreme point identified in Figure 3. At the same time, it is understandable, that such a simple scheme cannot easily be used to predict El Niño occurrence, because the duration of the SML cycles is irregular and the El Niños have a tendency to occur about the middle of the SML cycle (see Fig. 4). As a result, a time lag between the SML extreme point and the El Niño event will be different for different events. It is clear that more investigation is needed to find a reliable scheme for El Niño prediction based upon the list of SSC occurrence. 


\section{Conclusion}

The geographic longitude of the subsolar meridian (SML) evaluated at the time of geomagnetic Storm Sudden Commencements (SSCs) from 1968 to 2003 shows organized variations in time, rather than the initially expected random behavior. The longer-term (22-year) variation of the SML values is closely related to 22-year magnetic solar cycle. The shorter-term SML variations are characterized by quasiperiodic cycles of duration from about 3 to more than 6 years. These cycles are found to be closely coincident with climate ENSO-cycles: one El Niño event occurs in each SML cycle. An analysis based on SSC and El Niño records from 1968 to 2003 shows that on the average an El Niño event starts about 15-16 months after the beginning of the SML cycle. It is clear that much work remains to further verify this speculative assertion and to find the physical processes linking geomagnetic SSCs with climatic phenomena like El Niño.

Acknowledgements. We are very appreciated to P. Fabian and J. L. Santos and two anonymous referees who have edited and evaluated our paper. We wish to thank K. Wolter from the NOAA Climate Prediction Center for providing the monthly MEI values. Support for the work by G. Khachikjan during a visit to the Univ. of Saskatchewan was provided by an NSERC Canada Team Discovery Grant to G. J. Sofko and A. V. Koustov.

Edited by: P. Fabian and J. L. Santos

Reviewed by: two anonymous referees

\section{References}

Akasofu, S. I.: Energy coupling between the solar wind and the magnetosphere, Space Rev., 28, 121-190, 1981.

Berlage, H. P.: Fluctuations of the general atmospheric circulation of more than one year: Their nature and prognostic value, $\mathrm{K}$. Ned. Meteorol. Inst. Meded. Verh., 69, 152 p., 1957.

Bjerknes, J.: A possible response of the atmospheric Hadley circulation to equatorial anomalies of ocean temperature, Tellus, 18, 820-829, 1966.

Chernosky, E. J.: Double sunspot-cycle variation in terrestrial magnetic activity, 1884-1963, J. Geophys. Res., 71, 965-974, 1966.

Cliver, E. W., Kamide, Y., and Ling, A. G.: Mountains versus valleys: Semiannual variation of geomagnetic activity, J. Geophys. Res., 105, 2413-2424, 2000.

Gnevishev, M. N.: On the 11-years cycle of solar activity, Solar Phys., 1, 109-120, 1967.

Gonzalez, A. L. C. and Gonzalez, W. D.: Periodicities in the interplanetary magnetic field polarity, J. Geophys. Res., 92, 43574375, 1987.

Ferraro, V. C. A., Parkinson, W. C., and Unthank, H. W.: Sudden commencements and sudden impulses in geomagnetism, J. Geophys. Res., 56, 177-195, 1951.

Frohlish, C. and Lean, J.: The Sun's total irradiance: cycles, trends and related climate change uncertainties since 1976, Geophys. Res. Lett., 25, 4377-4380, 1998.

Hildebrandsson, H. H.: Que ques recherches sur les centres d'action de l'atmospehre, Kon. Sevenska Vetens. Akad. Handl., 29, 33 pp., 1897.
Jackson, W.: World-wide simultaneous magnetic fluctuations and their relation to sudden commencements, J. Atmos. Ter. Phys., 2, 160-172, 1952.

Kane, R. P.: Quasi-biennial and quasi-triennial oscillations in geomagnetic activity indices, Ann. Geophys., 15, 1581-1594, 1997.

Khachikjan, G., Sofko, G., Koustov, A., and Benkevitch, L.: Dependence of the occurrence frequency and time of storm sudden commencements on heliogeophysical conditions, Geomagnetism and Aeronomy, 45, 6, 720-729, 2005.

Khachikjan, G. and Petelin, A.: Prediction of El Niño phenomenon using space weather data, available at: www.cosis.net/ abstracts/COSPAR02/01761/COSPAR02-A-01761, last access: 30 November 2002.

Landscheidt, T.: Solar activity controls El Nino and La Nina, http://www.geocities.com/CapeCanaveral/4310/klima/elnino/ sun-enso.htm, 12 January 1999.

Lindsay, G. M., Luhmann, J. G., Russell, C. T., and Gaziz, P. R.: On the sources of interplanetary shocks at $0.72 \mathrm{AU}, \mathrm{J}$. Geophys. Res., 99, 11-17, 1994.

Mayaud, P. N.: Analysis of storm sudden commencements for the years 1868-1967, J. Geophys. Res., 80, 111-122. 1975.

Mayaud, P. N.: A 100-year series of geomagnetic data: Indices $a a$, storm sudden commencements, IAGA Bull. 33, 252 pp., Intern. Union of Geod. and Geophys., Paris, 1973.

Mendoza, B., Perez-Enriquez, R., and Alvarez-Madrigal, M.: Analysis of solar activity conditions during periods of El Nino events, Ann. Geophys., 9, 50-54, 1991.

Mock, D. R.: The Southern Oscillation: Historical Origins. University of Washington, Seattle, WA, 9 p., available at: http://www. cdc.noaa.gov/ENSO/Misc/hxsoi.html, last access: 28 September 2004, 1981.

Nielsen, E. and Honary, F.: Observation of ionospheric flows and particle precipitation following a Sudden Commencement, Ann. Geophys., 18, 908-917, 2000.

Pudovkin, M. and Veretenenko, S.: Cloudiness decreases associated with Forbush decreases of galactic cosmic rays, J. Atmos. Terr. Phys., 57, 1349-1355, 1995.

Quinn, W. H., Neal, V. T., and Antunez de May Gonzalez, S. E.: El Nino occurrences over the past four and a half centuries, J. Geophys. Res., 92, 14 449-14 461, 1987.

Ramage, C. S.: El Nino, Scientific American, 254, 77-83, 1986.

Reid, G. C.: Solar total irradiance variations and the global sea surface temperature record, J. Geophys. Res., 96, 2835-2844, 1991.

Russell, C. T. and McPherron, R. L.: Semi-annual variation of geomagnetic activity, J. Geophys. Res., 78, 92-108, 1973.

Russell, C. T., Ginskey, M., and Petrinec, S. M.: Sudden impulses at low-latitude stations: Steady state response for northward interplanetary magnetic field, J. Geophys. Res., 99, 253-261, 1994a.

Russell, C. T., Ginskey, M., and Petrinec, S. M.: Sudden impulses at low-latitude stations: Steady state response for southward interplanetary magnetic field, J. Geophys. Res., 99, 13 403-13 408 , 1994b.

Schell, I. I.: On the nature of the southern oscillation, J. Meteor., 13, 592-596, 1956.

Siscoe, G. L., Formisano, V., and Lazarus, V.: A calibration of the magnetopause, J. Geophys. Res., 73, 4869-4874, 1968.

Svensmark, H. and Friis-Christensen, E.: Variation of cosmic ray flux and global cloud coverage, a missing link in solar-climate relationships, J. Atmos. Sol. Terr. Phys., 59, 1225-1232, 1997.

Su, S. Y. and Konradi, A.: Magnetic Field Depression at the Earth's Surface Calculated From the Relationship Between the Size of the Magnetosphere and the Dst values, J. Geophys. Res., 80, 
195-199, 1975.

Vagina, L. I., Sergeev, V. A., Baker, D. N., and Singer, H. J.: Use of mid-latitude magnetic data for modeling and diagnostics of magnetospheric substorms, Adv. Space Res., 18, 8229-8232, 1996.
Walker, G. T.: World Weather, Mon. Wea. Rev., 56, 167-170, 1928. 